\title{
Base Report
}

\section{Management of penetrating zone II neck injuries}

\author{
Vivek Sasindran · Antony Joseph
}

\begin{abstract}
The incidence of penetrating and lacerated neck injuries has been on the rise recently because of the increase in road traffic mishaps. Clothesline injuries are injuries caused by striking against a stationery object like a wire or tree limb. Most of the times these end up causing blunt injuries with associated fracture of the laryngeal framework. Here we report two cases of penetrating neck injuries with successful outcomes and also the problems encountered in the management of these cases. A proper evaluation, rapid airway intervention and proper surgical repair are essential for a successful outcome.
\end{abstract}

Keywords Neck injuries. Penetrating wounds . Intubation

V. Sasindran · A. Joseph

Department of ENT,

Pushpagiri Medical College, Thiruvalla, Kerala.

V. Sasindran ( $ه)$

E-mail: viveksasindran@hotmail.com

\section{Introduction}

Penetrating neck injuries are potentially dangerous and require emergency treatment because of the presence of vital vessels and nerves in this region [1]. The exact documentation of these injuries can be useful to determine whether the injury was homicidal or self-inflicted [2]. Thorough knowledge of the anatomy of neck, physical assessment and diagnostic and therapeutic interventions are necessary for appropriate management.

\section{Case report 1}

A 28-year-old man was brought to our emergency department following an alleged road traffic mishap. A cable wire entangling around the neck leading to a cut throat injury while riding a bike. This type of injury commonly referred to as clothesline injury is a rarity these days. On examination a lacerated wound $10 \mathrm{~cm} \times 5 \mathrm{~cm}$ was noted in the upper part of the neck. The thyroid cartilage was exposed and the thyrohyoid membrane as completely transected (Fig. 1) exposing the pyriform fossa, posterior pharyngeal wall, arytenoids, aryepiglottic folds and the vocal cords. Externally, the mobility of the vocal cords could be appreciated on phonation. The hyoid bone and the epiglottis were also exposed. Patient was taken up for tracheostomy and neck exploration under general anesthesia. A translaryngeal intubation (Fig. 2) was followed up with a tracheostomy under general anesthesia. Endoscopic assessment revealed transected mucosa over both the pyriform fossae. Cricoarytenoid joints and the mucosa over the posterior pharyngeal wall were intact. Mucosa over the pyriform fossa was repaired in layers. Postoperatively patient was on Ryles tube feeds for 10 days. On the 11th day a test feed was given and the patient had aspiration. Patient was weaned off tracheostomy tube and allowed to take liquid diet by modified swallowing technique. On the 20th 


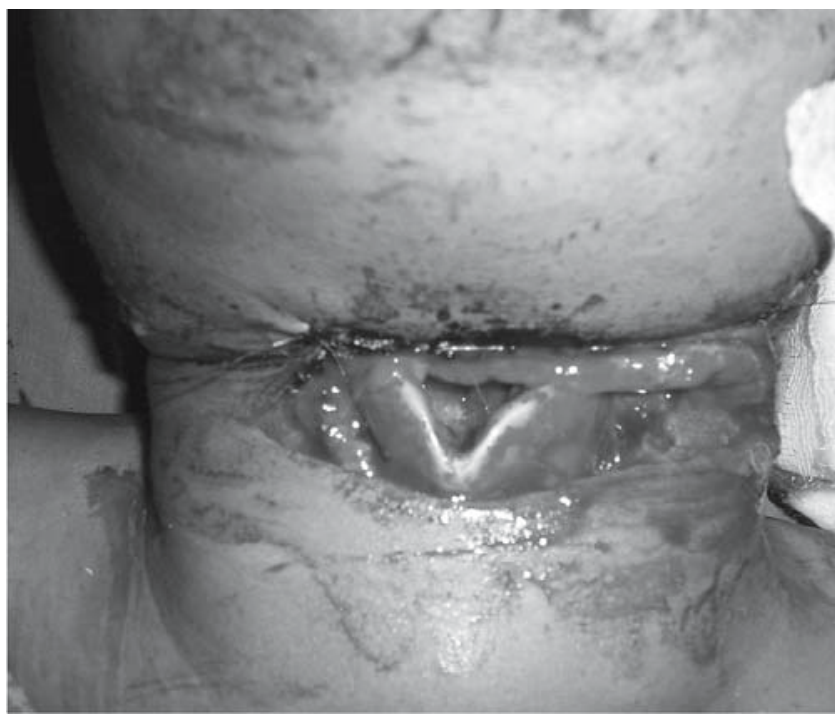

Fig. 1 Showing the transected thyrohyoid membrane and exposed thyroid cartilage

postoperative day patient had tenderness and discharge over the wound area with erythematous changes over the skin suggestive of early pharyngocutaneous fistula. Patient was continued on Ryles tube feed for another 5 days. Antibiotics were continued as per culture sensitivity reports. A week later he was able to take oral feeds without aspiration and the Ryles tube was removed.

Puffiness of the face and swelling over the limbs were noted and his renal functions showed a raised urea and creatinine. Nephrology opinion was sought for the same and he was diagnosed to have non-oliguric renal failure. Over the subsequent days the urea and creatinine levels progressively increased and the patient underwent hemodialysis for 3 consecutive days and his renal functions were back to normal. He was discharged from the hospital on the 42nd postoperative day and has been followed up for 3 months.

\section{Case report 2}

A 30-year-old male was brought to the casualty with alleged history of head on collision with a three wheeler and a piece of glass piercing the neck. There was no evidence of respiratory distress. On examination a $5 \mathrm{~cm} \times 3 \mathrm{~cm}$ horizontal laceration at the level of the cricothyroid membrane extending to the anterior border of the sternocleidomastoid muscle on either side with ragged skin margins (Fig. 3), oblique fracture line along the left lamina of the thyroid cartilage and inferior portion of the left thyroid lamina was missing. Horizontal fracture line along the upper border of the cricoid cartilage was seen. There was a defect in the cricothyroid membrane and the posterior lamina of the cricoid cartilage could be seen. There were multiple lacerations over the face and

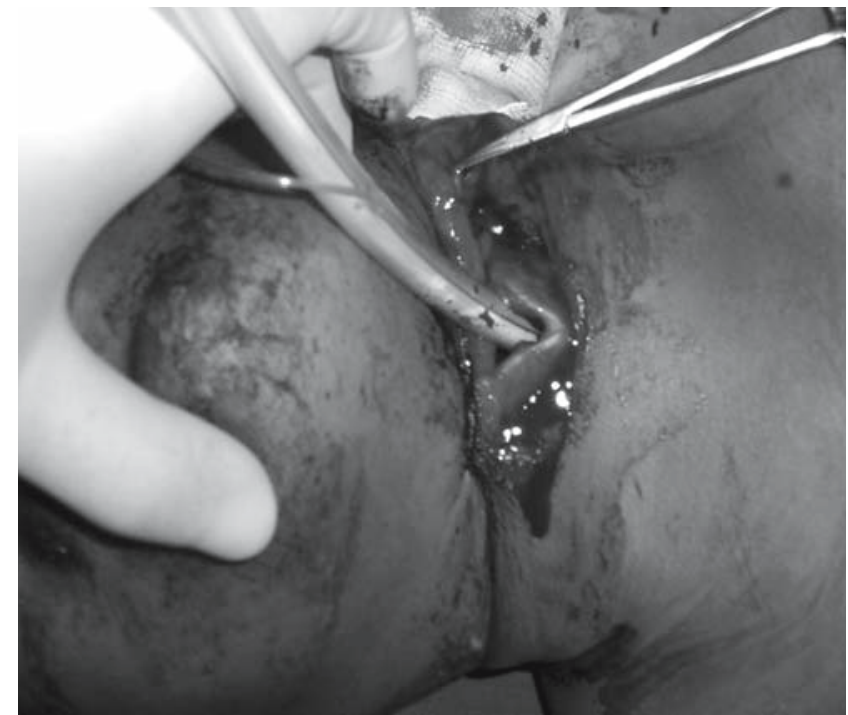

Fig. 2 Intubation through the neck wound

chest. Crepitus was palpable around the neck. CT scan of the neck revealed defect in the cartilage with subcutaneous emphysema (Fig. 4). Patient was taken up for exploration of the neck wound under general anesthesia. A tracheostomy was performed under local anesthesia to secure the airway and also for anesthesia. $30^{\circ}$ endoscope used for visualizing the subglottis and also the lower tracheal rings. The subglottis was clear of any injury. Cruciate shaped crack was seen over the inner perichondrium of the lamina of the cricoid cartilage. The inner perichondrium of the cricoid cartilage was sutured with vicryl. The thyroid and the cricoid cartilage were approximated and the wound closed. On the 7th postoperative day tracheostomy tube was removed. Endoscopy through the tracheal stoma showed the well healed mucosa. A Montgomery T-tube was inserted and dressings applied. Patient was discharged on the 10th postoperative day. At time of discharge patient was phonating well. Patient is on regular follow up with no complaints. Six months later patient was decannulated, the T-tube removed and the tracheal stoma closed. Patient has been followed up for a period of 6 months.

Penetrating neck injuries are usually described in terms of their location in one of the three anatomic zones as described by Monson [4].

Zone I is the region that extends from the inferior border of the cricoid cartilage to the clavicles.

Zone II is the area between the cricoid cartilage and the angle of the mandible.

Zone III comprises the area between the angle of the mandible and the base of the skull. 


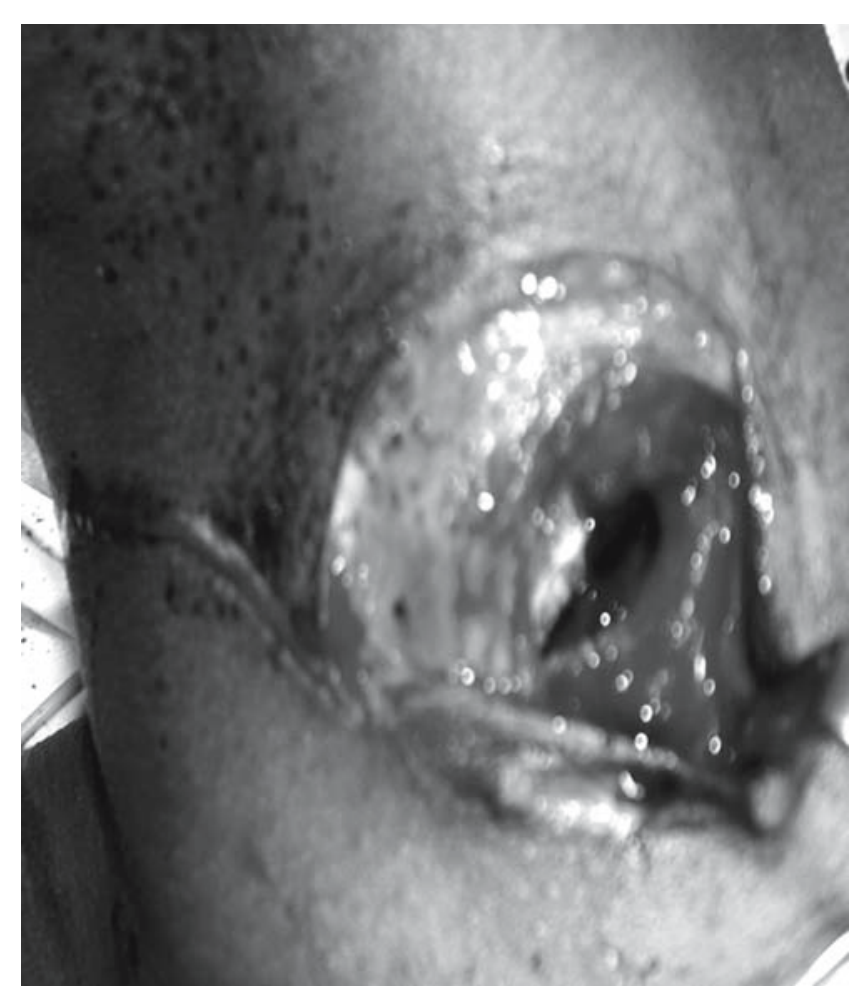

Fig. 3 Transection at the level of the cricothyroid membrane

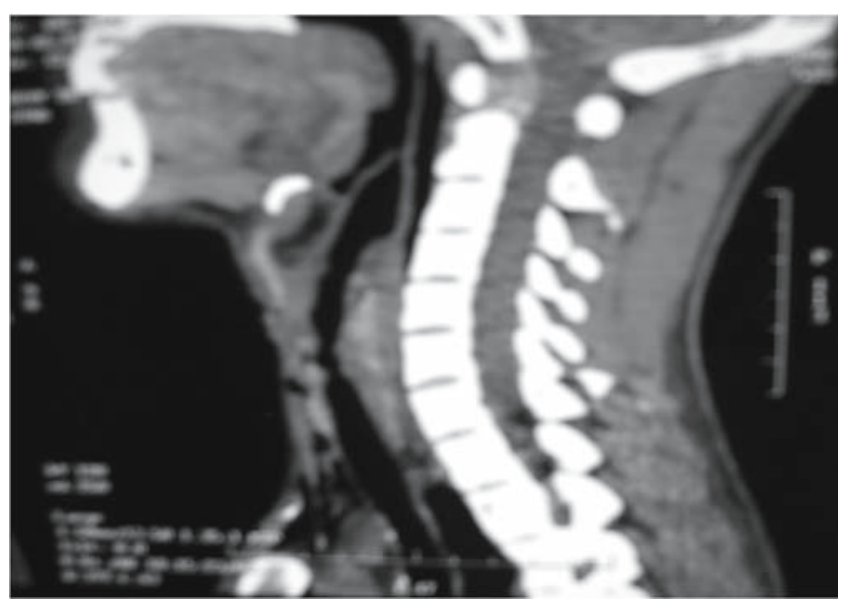

Fig. 4 Showing defect at the level of the cricoid cartilage with subcutaneous emphysema

\section{Discussion}

Penetrating neck injuries are seen in $5-10 \%$ of the trauma cases [1]. Penetrating wounds are caused by knives, bullets, wires and agricultural implements. Fogelman and Stewart in 1956 reported a mortality rate of $6 \%$ with prompt exploration versus $35 \%$ in cases with delayed or omitted operation. This led to widespread theory of treating the platysma like peritoneum: if violated explore. Later during the World War II, management changed to mandatory exploration of all neck wounds [3].
In both our cases the injuries were confined to zone II. This is the most frequently injured region in the neck. The mortality rate from these injuries is relatively low because of the better surgical exposure compared to the other zones.

It would be usual for a penetrating instrument to slide off the thyroid cartilage and penetrate the thyrohyoid membrane superiorly or go between the cricoid and thyroid inferiorly to penetrate the cricothyroid membrane. Penetration of the thyrohyoid membrane causes bleeding in the paraglottic space and thus airway obstruction. In case of cricothyroid membrane penetration air will leave the respiratory tract and will cause surgical emphysema in the neck as was seen in our second case. The bleeding may fill the subglottic space causing respiratory obstruction. The fate of the thyroid cartilages will depend upon the degree of calcification. Cricoid cartilage is the most important part of the laryngeal skeleton. It is the only complete ring in the upper and lower respiratory tract. If the cricoid is disrupted then it will stenose. Even a linear fracture in the cricoid will cause some resorption of the cartilage and reduction in the calibre of the airway at the level of the cricoid. CT scan should be performed to determine the integrity of the laryngeal framework.

The first and the foremost concern in a patient with a laryngeal framework injury is securing the airway. Injury to the cervical spine should be suspected in these patients until proven otherwise. There is some controversy over airway management in these patients, but most authors recommend tracheostomy under local anesthesia for patients exhibiting respiratory distress. Attempts at oral or nasotracheal intubations in these patients may result in further damage to an already tenous airway. A blind attempt at intubating the trachea may carry the risk of introducing the tracheal tube into a false passage or cause a complete disruption of the endolaryngeal structures, or even facilitating laryngotracheal separation [5]. In stable patients, flexible fiber optic laryngoscopy should be performed.

Laryngeal injuries may be treated medically or surgically depending on the initial fiber optic laryngoscopic and CT findings. Closed injuries can be managed with a minimum 24 hours of close observation, head end elevation, voice rest and humidified air. Antibiotics are recommended with disruption of laryngeal mucosa. Systemic steroids are given to reduce laryngeal edema. Nasogastric tube feedings should be considered to give rest to the laryngeal framework. Penetrating traumas require open exploration. When laryngeal exploration is indicated, it should be performed within 24 hours of the injury in order to maximize airway and phonation results [6]. Fractures of the cartilages are reduced and can be stabilized using a variety of materials, including stainless steel wires, non-absorbable suture and miniplates. If the fracture is comminuted, small fragments of cartilage with no intact perichondrium are removed to prevent chondritis. Endolaryngeal stenting is reserved for 
wounds involving disruption of the anterior commissure, massive mucosal injuries and comminuted fractures of the laryngeal skeleton. Stenting stabilizes severely comminuted fractures and prevents web formation and stenosis. The most common indication for laryngeal stenting follows reconstruction of laryngotracheal stenosis. Occasionally, laryngeal stents are used following trauma to larynx resulting in laryngeal fracture or injury. Stenting may help maintain lumen patency and prevent mucosal lacerations from scarring. The Montgomery T-tube used in our second case, is a device used as a combined tracheal stent and an airway. The tube can be used in acute tracheal injury, as a stent after tracheal reconstruction, and as a substitute for the non-reconstructable cervical trachea. The other commonly used stents are Aboulker stent, Silastic sheet (Swiss roll), Silicone stents, Inflatable stents and metal stents.

\section{Conclusion}

Management of laryngeal injury within 24 hours gives the best result for airway and voice. Aggressive surgery is indicated to prevent the complications such as hoarseness, laryngeal stenosis and dysphagia. Successful outcomes can be expected in patients in whom proper surgical repair has been done.

\section{References}

1. Ozturk K, Keles Bahar, Cenik Z, Yaman H(2006) Penetrating zone II neck injury by broken windshield. Int Wound $\mathrm{J}$ 3(1):63-66

2. Godbole BG, Vira TM, Rao RV (1980) Stab injury of neck. J Postgrad Med 26:257-258.

3. Vishwanatha B, Sagayaraj A, Huddar GH(2007) Penetrating neck injuries. Indian J Otolaryngol Head Neck Surg 59:221224

4. Desjardins G, Varon AJ (2001) Airway management for penetrating neck injuries: the Miami experience. Resuscitation 48:71-75

5. Verschueren DS, Bell RB, Bagheri SC, Dierks EJ, Potter BE (2006) Management of laryngotracheal injuries associated with cranio maxillofacial trauma. J Oral Maxillofacial Surg 64:203-214

6. Leopold DA (1983). Laryngeal trauma: A historical comparison of treatment methods. Arch Otolaryngol 109:106-110 